\title{
Cost-Effective TSV Grouping for Yield Improvement of 3D-ICs
}

\author{
Yi Zhao, Saqib Khursheed, Bashir M. Al-Hashimi \\ School of Electronics and Computer Science \\ University of Southampton, UK \\ Email: \{yz2g08,ssk, bmah\}@ecs.soton.ac.uk
}

\begin{abstract}
Three-dimensional Integrated Circuits (3D-ICs) vertically stack multiple silicon dies to reduce overall wire length, power consumption, and allow integration of heterogeneous technologies. Through-silicon-vias (TSVs) which act as vertical links between layers pose challenges for 3D integration design. TSV defects can happen in fabrication process and bonding stage, which can reduce the yield and increase the cost. Recent work proposed the employment of redundant TSVs to improve the yield of 3D-ICs. This paper presents a redundant TSVs grouping technique, which partitions regular and redundant TSVs into groups. For each group, a set of multiplexers are used to select good signal paths away from defective TSVs. We investigate the impact of grouping ratio (regular-to-redundant TSVs in one group) on trade-off between yield and hardware overhead. We also show probabilistic models for yield analysis under the influence of independent and clustering defect distributions. Simulation results show that for a given number of TSVs and TSV failure rate, careful selection of grouping ratios lead to achieving $100 \%$ yield at minimal hardware cost (number of multiplexers and redundant TSVs) in comparison to a design that does not exploit TSV grouping ratios.
\end{abstract}

Keywords-TSV, defect, yield, 3D-IC.

\section{INTRODUCTION}

Three-dimensional Integrated Circuits (3D-ICs) is a promising technology to overcome performance bottleneck of traditional integrated circuits due to higher interconnect delay [1]. 3D-ICs consists of stacking of multiple silicon dies with vertical interconnects between them. There are a number of reported technologies (IBM [2], IMEC [3], MIT [4]) that implement 3D integration. Through-silicon-vias (TSVs) based $3 \mathrm{D}$ integration is one of the most promising technologies, which can stack wafers or dies with vertical TSV interconnects. Using TSVs technology, a very high interconnects density, millions of TSVs in a design, can be realized [5]. However, yield of TSVs based 3D-ICs is limited under current manufacturing process. Only one defective TSV can fail the entire chip with all known-good dies [6].

Redundant circuits can be an efficient solution to improve the yield of 3D-ICs. For example, [7] increases the yield of 3D-stacked memory by sharing the redundant memory rows/columns across neighbouring dies. [8] attempts to improve the yield by providing wireless redundant TSVs. [9] proposes a fault tolerance scheme based on redundant TSVs and multiplexers, to ensure availability of good signal paths between layers by rerouting signals through non-defective redundant TSVs. [10] looks into repair mechanism, considering designs with small number of TSVs (up to 500) and partitions TSVs into blocks and assigns each block with one spare TSV for repairing the faulty link in that block. In [11], [12], similar solutions are proposed to improve the yield of 3D-ICs. This work is also based on utilizing redundant TSVs, we partition regular and redundant TSVs into groups using a specified grouping ratio (regular-to-redundant), where each group can have multiple spare TSVs, and multiplexers are used to reroute signals through good TSV path in case defective TSVs exist in that group. Clustering defects have been acknowledged in traditional semiconductor manufacturing to cluster in an area rather than randomly distributed, for memories clustering defects have been reported in literature [13], [14]. Clustering defects are also considered in this work to analyse their affect on yield of 3DICs. To the best of our knowledge, this is the first study to model clustering defects and analyse yield in the presence of clustering defects in 3D-ICs. Simulation results evaluate the trade-off between yield and hardware cost (number of multiplexers and spare TSVs) under the influence of independent and clustering defect distributions, and show that it is possible to achieve $100 \%$ yield at minimal hardware cost through careful selection of grouping ratios and redundant TSV percentage.

This paper is organized as follows: Section 2 use an example to illustrate the problem examined in this paper. Section 3 presents the TSV redundancy modeling methodology and the yield analysis approach. Section 4 presents simulation results by exploring the yield of a number of regular TSVs under different grouping ratios. Finally, section 5 concludes this paper.

\section{Motivation AND PROBLEM FORMULATION}

\section{A. Motivation}

The manufacturing process of TSVs based 3D-ICs can be summarized into three stages. Firstly, the fabrication of individual dies to be stacked, which involves transistor layer and metal layers construction. Secondly, the fabrication processing of TSV, which involves via etching and filling procedures [3]. Finally, the bonding stage, which bonds TSV with the bonding pad to form the communication link between dies. These steps can also be re-ordered to build TSVs before transistors and metal layers. Misalignment and random open defects are two main TSV failure mechanisms [9]. Firstly, 
random open defects can happen in TSV fabrication process, due to processing variants such as insufficient filling, voids formation, etc. Similarly in bonding process, random open defects may be caused by foreign particles [15]. Secondly, misalignment is due to incorrect wafer alignment during bonding, which results in shift of TSV tips with their bonding pads. Misalignment can be addressed by increasing the bonding accuracy [16], and therefore we focus on random open defects in this paper.

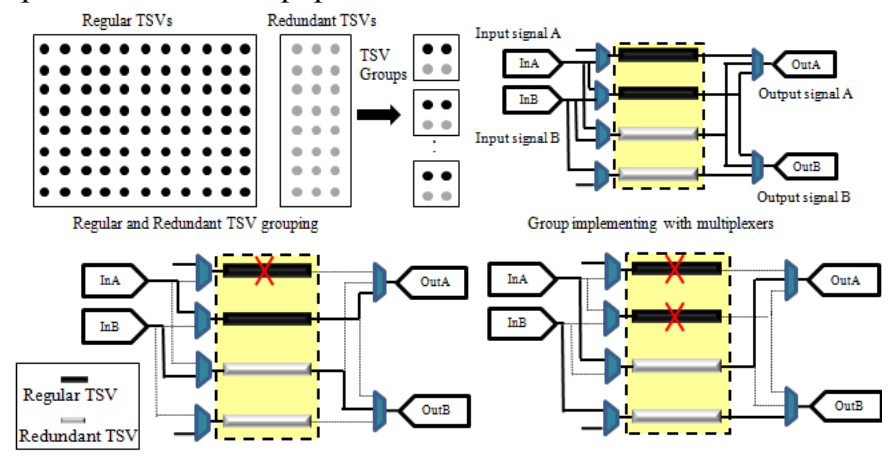

Fig. 1 Motivation of grouping technique

Figure 1 illustrates the basic idea of our proposed grouping technique, for a number of regular TSVs, we provide redundant TSVs. Then we partition them into groups according to grouping ratio $\left(g r=N_{g r}: N_{g s}\right)$ which accounts for the number of regular TSVs $\left(N_{g r}\right)$ and redundant TSVs $\left(N_{g s}\right)$ to be placed in a group. Multiplexers are used to select good signal paths avoiding the defective TSVs. As an example see Figure 1, where each group contains two regular and two redundant TSVs, we could repair the group in case of one or two faulty TSVs through multiplexer rerouting. Then we illustrate how redundant TSVs with regular ones have an impact on the yield and hardware cost (redundant TSVs and multiplexers). Assuming we have eight regular TSVs and four spare TSVs in total, which can be organized in two grouping ratios, as shown in Figure 2.

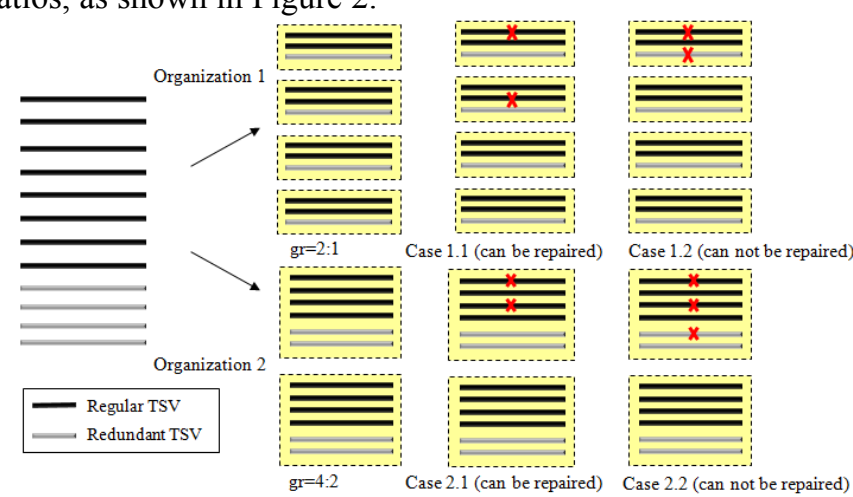

Fig. 2 Eight regular and four redundant TSVs partitioned by grouping ratio (Regular: Redundant) 2:1 and 4:2

- Organization 1: Grouping ratio is 2:1. Four groups are obtained, with two regular TSVs and one redundant TSV in each group.

- Organization 2: Grouping ratio is 4:2. Two groups are obtained, each with four regular and two redundant TSVs.

Organization 1 allows maximum one defective TSV within a group, such that the group can be repaired (case 1.1). If two defective TSVs are found within one group, such as in case 1.2 the group cannot be repaired. However, with the same redundancy percentage, organization 2 tolerates maximum two defective TSVs in one group, which indicates that, if only two defective TSVs exist, organization 2 can always be repaired (case 2.1). If more than two defective TSVs exist in one group (case 2.2), organization 2 cannot be repaired. It is clear that higher grouping ratio (4:2) implies higher yield. The cost of our grouping technique involves spare TSVs and multiplexers. Figure 3 illustrates the multiplexer configurations for both grouping ratios 2:1 and 4:2, and summarizes the multiplexer cost in the Table. Although grouping ratio 4:2 implies higher yield, it requires higher multiplexer cost in terms of area overhead. We need to evaluate how redundant TSVs should be grouped with regular ones to achieve the best yield with the lowest possible hardware cost (redundant TSVs and multiplexers) at a given fault rate. In terms of placement of timing critical signals, we use the method proposed in a recent study [10] that shows a timing-aware TSV placement method such that if signal rerouting is required due to defective TSV in a group then the most timing critical signal is least affected. See [10] for more details
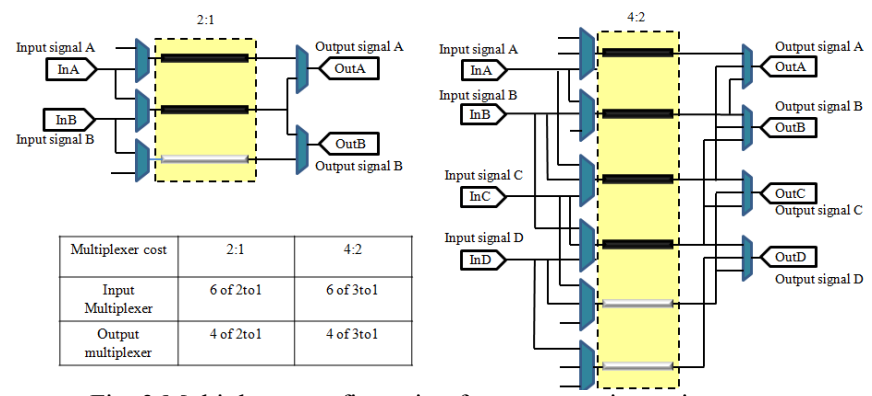

Fig. 3 Multiplexer configuration for two grouping ratios

\section{B. Problem formulation}

The problem addressed in this paper can be formulated as: Problem: Given

- $\quad$ The number of regular TSVs $N_{R}$;

- $\quad$ The failure rate of a single TSV $p$;

Analyse the yield under different grouping ratios (regular: redundant TSVs in one group) and attempt to achieve a target yield with the lowest possible hardware cost (redundant TSVs and multiplexers). The best grouping ratios to achieve highest yield and lowest hardware cost is determined through an exhaustive search across all possible grouping ratios until $100 \%$ yield is achieved. This is further explained in Sec. 5.

To solve this problem, we first investigate how to model the TSV redundancy, and then we use a probabilistic method to achieve the yield in the presence of independent and clustering defects.

\section{TSV REDUNDANCY MODELLING AND YIELD ANALYSIS}

In this section, we first consider each TSV to have independent failure rate. TSV redundancy modelling for independent defect distribution, partition regular and redundant TSVs into groups, and the overall yield $Y_{\text {independent }}$ 
can be obtained based on a probabilistic model. Next, we introduce clustering defects when modelling TSV redundancy which means that the defective probability of a TSV increases due to existing defects (defect correlation). TSV location is required in this model and we propose an algorithm to calculate the yield of TSV redundancy for clustering defect distribution, denoted by $Y_{\text {clustering. }}$.

\section{A. TSV redundancy modeling and analysis for independent defect distribution}

For independent defect distribution based TSV redundancy model, the basic modelling features are (1) redundancy percentage $r d$ is the usage of redundant TSVs (Redundancy/Regular), (2) grouping ratio, regular to spare TSVs ratio $\left(N_{g r}: N_{g s}\right)$ in a group. The total number of spare TSVs is $N s=N r^{*} r d$. All TSVs are then partitioned into groups according to grouping ratio, the number of groups is given by $g n=\left[\frac{\mathrm{N}_{\mathrm{r}}}{\mathrm{N}_{\mathrm{gr}}}\right]$. The post-partition groups are denoted by $G_{l}, \cdots, G_{i}$, $\cdots, G_{g n}$. The uniform group size is $\left|G_{i}\right|=N_{g r}+N_{g s}$. Each group is independent from each other. If we achieve the yield of one group $Y_{\text {group }}$, then the overall yield of all gn groups $Y_{\text {independent }}$ can be obtained by multiplying all individual group yields, expressed as

$$
Y_{\text {independent }}=\left(Y_{\text {group }}\right)^{g n}
$$

Each TSV within a group is independent and has a uniform failure rate $p$. Thus, the number of defective TSV in a group follows binomial distribution [10], which is: Assuming $X$ is the variable of defective TSV number in a group, then the probability of having $x$ defective TSVs is expressed as

$$
P(\mathrm{X}=x)=C_{N_{g r}+N_{g s}}^{x} \cdot p^{x} \cdot(1-p)^{\left(N_{g r}+N_{g s}-x\right)}
$$

where $C_{N_{g r}+N_{g s}}^{x}$ is a combination of $x$ and $\left(N_{g r}+N_{g s}\right)$ which shows all the possible situations of having $x$ defective TSVs in a group of $\left(N_{g r}+N_{g s}\right)$ TSVs. Clearly if defective TSVs number in a group is smaller than the spare TSVs number $N_{g s}$, such group can be repaired. Therefore, the yield of one group $Y_{\text {group }}$ is

$$
Y_{\text {group }}=\sum_{x=0}^{N_{g s}}\left[C_{N_{g r}+N_{g s}}^{x} \cdot p^{x} \cdot(1-p)^{\left(N_{g r}+N_{g s}\right)-x}\right]
$$

Equation 3 calculates the overall probability of having $x(0$ * $\left.\cdot N_{g s}\right)$ defective TSVs in a group. The yield of TSV redundancy for independent defect distribution can be achieved by substituting Eq. 3 into Eq. 1.

\section{B. TSV Redundancy Modelling and Yield analysis for cluster- ing defect distribution}

We consider clustering defects, where all TSVs are correlated, and therefore the modelling method is different from independent defect distribution (Eq. 1). This modelling scenario has to take TSVs location into accounts. We will first discuss the clustering effect before explaining the model.

Clustering defects means that defects tend to cluster together to some extent rather than randomly distributed. It models the scenario, where the presence of single defect increases the likelihood of more defects in close vicinity [17]. [18] described this clustering effect as defect probability of node $i\left(P_{i}\right)$ is inversely proportional to the distance from the existing defect node $j$, that is expressed as

$$
P_{\mathrm{i}} \propto\left(\frac{1}{d_{\mathrm{ij}}}\right)^{\alpha}
$$

where, $d_{\mathrm{ij}}$ indicates the distance between node $i$ and defective node $j$, and $\alpha$ is the clustering coefficient indicating clustering extent, a larger $\alpha$ implies higher clustering. By employing the concept 'cluster center' [19], in our paper a cluster center represents one defective TSV, where all defective TSV tend to exist around this center. The defective probability of $T S V_{i} P_{\mathrm{i}}$ will be increased, which can be expressed as

$$
P_{\mathrm{i}}=p \cdot\left(1+\left(\frac{1}{d_{\mathrm{ic}}}\right)^{\alpha}\right)
$$

where $p$ is the single TSV failure rate, $d_{\text {ic }}$ is the distance between $\operatorname{TSV}_{i}$ and cluster center. This is illustrated in Figure 4(a)-(c), where hollow node represents the cluster center defective TSV, solid nodes denote the other nearby defective TSVs. By taking clustering effect into consideration, the distribution of defective TSVs tends to cluster around a cluster center and this becomes higher with larger clustering coefficient.

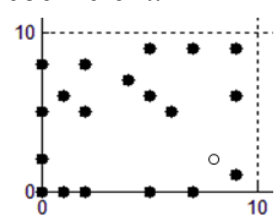

(a) No clustering

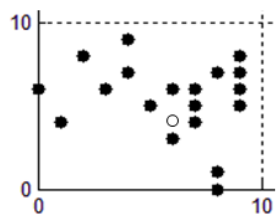

(b) $\alpha=1$

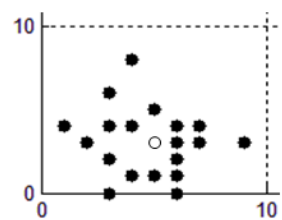

(c) $\alpha=2$
Fig. 4 Defective TSV pattern illustrating clustering effect

The clustering TSV redundancy model assigns all TSV groups $\left\{G_{1} \cdots G_{i} \cdots G_{g n}\right\}$ into 'blocks', each block refers to a wafer area that contains TSV groups. A defects cluster within a block is regarded as small cluster and each block can have one cluster. Therefore, each block is independent and clusters within different blocks do not affect each other [20], [21]. The size of block, namely how many TSVs in a block, is uniform denoted as $|Q|$, such that all groups $\left\{G_{1} \cdots G_{i} \cdots G_{g n}\right\}$ are located into $q n$ blocks, $q n=\frac{N_{r}+N_{s}}{|Q|}$. Each block is assigned with a $\mathrm{N} \times \mathrm{N}$ grid with $x$ and $y$ orientation coordinates range in $(0<=x<=\sqrt{|\mathrm{Q}|}-1,0<=y<=\sqrt{|\mathrm{Q}|}-1)$ respectively. We assume that each TSV is located on the integral coordinates, this regular placement scenario also comply with the fabrication processing. A block size $|Q|=100$ is used in our work as this size meets the requirement of having small size defects cluster in a block. Under this block setting, each $\mathrm{TSV}_{k}$ placed in this block has its unique properties denoted as $\mathrm{TSV}_{k}\left\{X_{k}, Y_{k}\right.$, group_index $\}$, where $k$ is the TSV subscript, $X_{k}$ and $Y_{k}$ are its horizontal and vertical coordinates respectively which will be used to calculate the distance between two

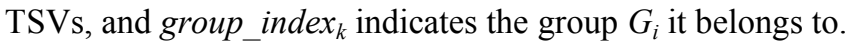

The overall yield of TSV redundancy for clustering defect distribution, denoted by $Y_{\text {clustering }}$ can be obtained by multiplying all individual block yield as

$$
Y_{\text {clustering }}=\left(Y_{\text {block }}\right)^{q n}
$$

If a block contains a defective TSVs cluster of which more than $N_{g s}$ (redundant TSV number in one group) defective 
TSVs are found in a group, then it cannot be repaired. Let $P_{\text {non-repair }}$ indicates the probability that a block cannot be repaired, the yield of a block expressed as

$$
Y_{\text {block }}=1-P_{\text {non-repair }}
$$

An algorithm is proposed to calculate $P_{\text {non-repair }}$ by identifying all the cases that a block contains a cluster with groups that cannot be repaired and sum up the probability of each case. Figure 7 shows the $P_{\text {non-repair }}$ calculating algorithm. It begins by TSV setup process (Step 1) which assigns coordinates to TSVs within block. TSVs are not randomly assigned a coordinate, for the sake of simplification, this is done in a group-by-group manner, once a group of TSVs are located then it deals with another group. An example of TSV placement in block is illustrated in Figure 5.

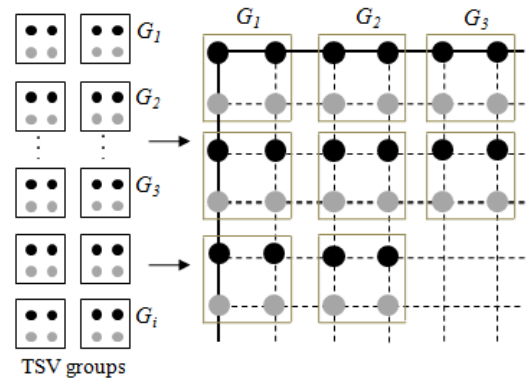

Fig. 5 TSVs are placed in the block group by group, grouping ratio=2:2, black node and grey node denotes regular and redundant TSVs respectively.

Next step is to find out all the possible situations that a block contains a cluster with more than one group which cannot be repaired (Step 2). To ensure that at least one group that cannot be repaired exists, the possible defective TSV number of one block, denoted by $N_{d}$, should be larger than $N_{g s}$. However, a block containing more than $N_{g s}$ defective TSVs may be repaired. A defective TSV pattern gives the defective TSV distribution in a block. Using block in Figure 5 as an example, if total defective TSV number in that block is four, two possible defective TSV patterns are $\left\{\left(\mathrm{TSV}_{1}, \mathrm{TSV}_{2}\right.\right.$, $\left.\left.\mathrm{TSV}_{3}\right),\left(\mathrm{TSV}_{5}\right)\right\}$ and $\left\{\left(\mathrm{TSV}_{1}, \mathrm{TSV}_{3}\right),\left(\mathrm{TSV}_{6}, \mathrm{TSV}_{7}\right)\right\}$, as shown

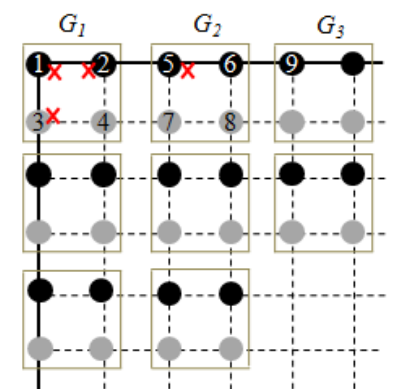

(a) $\left\{\left(\mathrm{TSV}_{1}, \mathrm{TSV}_{2}, \mathrm{TSV}_{3}\right),\left(\mathrm{TSV}_{5}\right)\right\}$

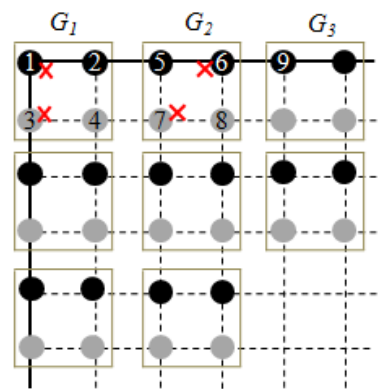

(b) $\left\{\left(\mathrm{TSV}_{1}, \mathrm{TSV}_{3}\right),\left(\mathrm{TSV}_{6}, \mathrm{TSV}_{7}\right)\right\}$
Fig. 6 Examples of defective TSV patterns

in Figure 6. Defective pattern $\left\{\left(\mathrm{TSV}_{1}, \mathrm{TSV}_{3}\right),\left(\mathrm{TSV}_{6}, \mathrm{TSV}_{7}\right)\right\}$ can be repaired, as no group contains more than two defective TSVs. All possible defective patterns that represent the case of a block that cannot be repaired are then stored into variable non_repair_pattern. Then we start calculating $P_{\text {non-repair }}$, by summing up the probability of each defective pattern in non_repair_pattern (Step 3-Step 8). All TSVs in a defective
Algorithm Probability of a block that cannot be repaired

1 Place TSVs into block, each $\mathrm{TSV}_{k}$ has its property $\mathrm{TSV}_{\mathrm{k}}\left(X_{k}, Y_{k}\right.$, group index $\left.k\right)$;

2 Find out all cases that a block cannot be repaired, which are stored in non_repair_pattern

3 Calculate $P_{\text {non-repair }}$ by sum up the probability of each defective TSV pattern from step 2

$4 P_{\text {non-repair }}=0$

5 for each pattern $\in$ non_repair_pattern do

// Calculating the probability of one defective TSV pattern

$6 \quad P_{\text {pattern }}=$ probability of a defective TSV pattern

$7 \quad P_{\text {non-repair }}=P_{\text {non-repair }}+P_{\text {pattern }}$

8 end for

9 Return $P_{\text {non-repair }}$

Fig. 7 Algorithm for calculating probability of a block that cannot be repaired

TSV pattern are divided into two parts, $N_{d}$ defective TSVs and $|Q|-N_{d}$ non-defective TSVs, which are denoted as defective_part and non_defective_part respectively. Based on the multiplication principle the probability of a defective pattern can be achieved by multiplying the probability of $N_{d}$ defective TSVs $\left(P_{\text {defective part }}\right)$ and $|Q|-N_{d}$ non-defective TSVs $\left(P_{\text {non defective part }}\right)$. Also, as mentioned earlier, a cluster center refers to a defective TSV, and its distribution is uniform, indicating any defective TSV could be cluster center. If the cluster center varies, the defective probability of each TSV changes which results in different $P_{\text {defective part }}$ and $P_{\text {non_efective part }}$ Therefore, the probability of a defective TSV pattern contains $N_{d}$ defective TSV is expressed as

$$
\sum_{i=1}^{N_{d}}\left(P_{\text {defective_part }} \cdot P_{\text {non_defective_part }}\right)_{i} \cdot \frac{1}{N_{d}}
$$

where $i=\left(1 \cdots N_{d}\right)$ implies each defective TSV has been considered to be the cluster center, ' $\frac{1}{N_{d}}$, implies that cluster center follows uniform distribution and the probability equals to $1 / N_{d}$. The probability of $N_{d}$ defective TSVs $P_{\text {defective part }}$ is the product of defective probability of each defective TSV which is expressed as

$$
P_{\text {defective_part }}=p \cdot \prod_{m=1}^{N_{d}-1} \text { defective_prob }\left(T S V_{m}\right)
$$
where $p$ is the defective probability of cluster center and equals to TSV failure rate. 'defective_prob $\left(T S V_{m}\right)$ ' is the defective probability of defective $\mathrm{TSV}_{m}$ excluding cluster center, based on Eq. 5 , it equals to $p \cdot\left(1+\left(\frac{1}{d_{m}}\right)^{\alpha}\right)$, where $d_{m}$ is the distance between $\mathrm{TSV}_{m}$ and the cluster center.

Similarly, the probability of $|Q|-N_{d}$ non-defective TSVs $P_{n o n}$ defective part is the product of probability of the remaining non-defective TSVs which is

$P_{n o n \_ \text {defective_part }}=\prod_{n=1}^{|Q|-N_{d}}$ non_defective_prob $\left(T S V_{n}\right)$ where 'non_defective_prob $\left(T S V_{n}\right)$ ' denotes the probability of non-defective $\mathrm{TSV}_{n}$, which equals to $1-p \cdot\left(1+\left(\frac{1}{d_{n}}\right)^{\alpha}\right)$, and $d_{n}$ is the distance between $\mathrm{TSV}_{n}$ and the cluster center. 


\section{SIMULATION RESULTS}

Two sets of experiments are conducted to evaluate the performance of the proposed grouping technique of regular and redundant TSVs. The objective of both experiments is to maximize yield and minimize hardware cost (multiplexer and spare TSV), where TSV failure rate is a constraint. The objectives are achieved through careful selection of grouping ratios and redundancy percentage of spare TSVs. The first experiment examines the effect of grouping ratios on different number of regular TSVs affected by various TSV failure rates when considering independent defect distribution. The second experiment evaluates the trade-off between yield and hardware cost (multiplexer and spare TSV), when considering both independent and clustering defect distributions.

TABLE 1 Trade-off analysis between yield and hardware cost (multiplexers and spare TSVs) when considering independent defect distribution.

\begin{tabular}{|c|c|c|c|c|c|c|c|c|c|c|c|}
\hline $\begin{array}{l}\text { Grouping } \\
\text { ratio (gr) }\end{array}$ & \multicolumn{5}{|c|}{$5: 4$} & \multicolumn{6}{|c|}{$10: 4$} \\
\hline $\begin{array}{c}\text { Redunda- } \\
\text { ncy } \\
\text { percentage } \\
(\%)\end{array}$ & \multicolumn{5}{|c|}{80} & \multicolumn{6}{|c|}{40} \\
\hline $\begin{array}{c}\text { Number } \\
\text { of Regular } \\
\text { TSVs }\end{array}$ & \multicolumn{2}{|l|}{10,000} & \multicolumn{3}{|c|}{$1,000,000$} & \multicolumn{3}{|c|}{10,000} & \multicolumn{3}{|c|}{$1,000,000$} \\
\hline $\begin{array}{c}\text { TSV } \\
\text { failure } \\
\text { rate } p\end{array}$ & $\begin{array}{lll}0.001 & 0.005\end{array}$ & 0.01 & 0.001 & 0.005 & 0.01 & 0.001 & 0.005 & 0.01 & 0.001 & 0.005 & 0.01 \\
\hline Yield (\%) & \begin{tabular}{|l|l|}
100 & 100 \\
\end{tabular} & 100 & 100 & 100 & 100 & 100 & 100 & 100 & 100 & 99 & 98 \\
\hline $\begin{array}{l}\text { Spare } \\
\text { TSV }\end{array}$ & \multicolumn{2}{|l|}{8,000} & \multicolumn{3}{|c|}{800,000} & \multicolumn{3}{|c|}{4,000} & \multicolumn{3}{|c|}{400,000} \\
\hline $\begin{array}{c}\text { Multiplexer } \\
\text { (2-to-1) }\end{array}$ & \multicolumn{2}{|c|}{$1.12 \times 10^{5}$} & \multicolumn{3}{|c|}{$1.12 \times 10^{7}$} & \multicolumn{3}{|c|}{$9.6 \times 10^{4}$} & \multicolumn{3}{|c|}{$9.6 \times 10^{4}$} \\
\hline
\end{tabular}

In the first experiment, we analyse the effect of independent defect distribution on yield and hardware cost, where hardware cost is expressed in terms of spare TSVs and multiplexers. Yield and hardware cost is analysed by varying the number of regular TSVs and failure rates for two grouping ratios 5:4 and 10:4 respectively. These two grouping ratios are chosen for illustration purposes. The results are shown in Table 1 for 10,000 and $1,000,000$ regular TSVs with TSV failure rate of $0.001,0.005$, and 0.01 . The number of regular TSV is chosen because recent designs have millions of regular TSVs [5], [6]. The TSV failure rate is not exactly known and recent publications have chosen various failure rates from $10^{-4}$ [10] to 0.05 [8] and in this work it is up to 0.01 to account for increased TSV count in recent designs. In this case (independent defect distribution), yield is calculated by using Eq. 1 and Eq. 3. In Table 1, we analyze the two groups of regular TSVs $(10,000$ and $1,000,000)$ separately to analyze the trade-off between hardware cost and yield. In case of 10,000 regular TSVs, it can be seen that for all TSV failure rates ( 0.001 to 0.01$), 100 \%$ yield is achieved by both grouping ratios 5:4 and 10:4. However grouping ratio 5:4 requires higher hardware cost than the grouping ratio of $10: 4$, as it requires additional 4,000 spare TSVs and 16,000 multiplexers. This is because under binomial distribution and given failure rate (as input), grouping ratio of 10:4 gives enough spare TSVs in one group and more spare TSVs do not result in yield improvement. In case of 1,000,000 regular TSVs, failure rate starts to show its effects on yield. As can be seen, at TSV failure rate of $0.001,100 \%$ yield is achieved using grouping ratio of 10:4 (lower hardware cost), however this trend changes at higher failure rates $(0.005$ and 0.01$)$ and yield drops by up to $2 \%$. This means for $100 \%$ yield, grouping ratio of 5:4 is better than 10:4. This table clearly shows the tradeoff between yield and hardware cost and demonstrates that it is possible to achieve $100 \%$ yield at lower hardware cost by careful selection of grouping ratios. Grouping ratios and redundancy percentage is determined through an exhaustive search to achieve highest yield and lowest hardware cost as shown in the next experiment.

TABLE 2 Trade-off analysis between yield and hardware cost when considering independent and clustering defect distribution.

\begin{tabular}{|c|c|c|c|c|c|c|}
\hline \multirow{3}{*}{\multicolumn{2}{|c|}{$\begin{array}{c}\text { Redundancy } \\
\text { percentage }(\%)\end{array}$}} & \multirow{4}{*}{$\begin{array}{c}\text { Grouping } \\
\text { ratio } \\
(g r) \\
\\
1: 1 \\
\end{array}$} & \multicolumn{3}{|c|}{$\begin{array}{l}\text { Yield }(\%) \text { under Two types } \\
\text { of defect distributions }\end{array}$} & \multirow{4}{*}{$\begin{array}{c}\text { Multiplexer } \\
\text { cost } \\
(2-\text { to- } 1)\end{array}$} \\
\hline & & & \multirow{2}{*}{ Independent } & \multicolumn{2}{|c|}{ Clustering } & \\
\hline & & & & $\alpha=1$ & $\alpha=2$ & \\
\hline 1 & \multirow{5}{*}{$\begin{array}{c}100 \% \\
(6000 \\
\text { spare } \\
\text { TSVs) }\end{array}$} & & 55 & 31 & 7.0 & \\
\hline 2 & & $2: 2$ & 99 & 96 & 87 & 24000 \\
\hline 3 & & $3: 3$ & 100 & 100 & 99 & 42000 \\
\hline 4 & & $4: 4$ & 100 & 100 & 100 & 60000 \\
\hline 5 & & $5: 5$ & 100 & 100 & 100 & 78000 \\
\hline 6 & \multirow{5}{*}{$\begin{array}{c}50 \% \\
(3000 \\
\text { spare } \\
\text { TSVs) }\end{array}$} & $2: 1$ & 41 & 20 & 4.0 & 15000 \\
\hline 7 & & $4: 2$ & 97 & 91 & 79 & 30000 \\
\hline 8 & & $6: 3$ & 100 & 99 & 98 & 45000 \\
\hline 9 & & $8: 4$ & 100 & 100 & 100 & 60000 \\
\hline 10 & & $10: 5$ & 100 & 100 & 100 & 75000 \\
\hline 11 & \multirow{5}{*}{$\begin{array}{c}25 \% \\
(1500 \\
\text { spare } \\
\text { TSVs) }\end{array}$} & $4: 1$ & 23 & 9.0 & 1.0 & 13500 \\
\hline 12 & & $8: 2$ & 96 & 79 & 57 & 27000 \\
\hline 13 & & $12: 3$ & 99 & 98 & 94 & 40500 \\
\hline 14 & & $16: 4$ & 100 & 100 & 99 & 54000 \\
\hline 15 & & $20: 5$ & 100 & 100 & 100 & 67500 \\
\hline
\end{tabular}

In the second experiment, we analyze the trade-off between yield and hardware cost, when considering clustering defect distribution and it is shown that grouping ratios and redundancy percentages can be used to achieve $100 \%$ yield and reduce hardware cost, when considering clustering defects. In this case (clustering defect distribution) yield is calculated using the algorithm shown in Figure 7. For this experiment, we fix the number of regular TSVs and failure rate and for illustration purposes, we consider 6000 regular TSV and 0.01 failure rate. The simulation results are shown in Table 2. For this experiment, we consider three sets of redundancy percentages $(100 \%, 50 \%$ and $25 \%)$ as shown in the first column, and for each set of redundancy percentage, we consider five grouping ratios as shown in the second column. These five grouping ratios are used because the yield saturates at $100 \%$. Yield is calculated for two clustering coefficients $(\alpha=1$ and $\alpha=2)$ and for each clustering coefficient, the results are shown in the third column along with yield results for independent defect distribution for comparison. In this work, we analyze only two clustering coefficients and other clustering coefficients can be similarly analyzed. The last column shows 2-to-1 multiplexer overhead for each grouping ratio and redundancy percentage. 
From Table 2, we make the following three observations. Firstly, it can be seen that to achieve $100 \%$ yield, independent defect distribution incurs lower hardware cost in comparison to clustering defect distribution. For example, as shown in Table 2, when considering 100\% redundancy, independent defect distribution obtains $100 \%$ yield using a grouping ratio of 3:3, which requires 42,000 (2-to-1) multiplexers, while at $\alpha=2$ clustering coefficient, $100 \%$ yield is obtained by using $4: 4$ grouping ratio, which requires $(60,000-42,000) \quad 18,000$ additional multiplexers. This trend continues with the other two sets of redundancy percentages.

Secondly, when observing the yield drop across the two defect distributions (independent and clustering), it can be observed that in cases of small number (less than 2) of spare TSV per group, the yield drop is more drastic than groups with additional spares. For example, in case of $100 \%$ redundancy and grouping ratio of $1: 1$, the yield is only $31 \%$ and $7 \%$ in case of two clustering coefficients ( $\alpha=1$ and $\alpha=2)$, while at grouping ratio of $3: 3$, it is $100 \%$ and $99 \%$ when considering the two clustering coefficients. This is because in case of clustering defects, as shown in Fig. 4, the defect probability of a TSV increases if that is close to clustering center. The probability of a cluster with more than three defective TSVs is much smaller than a cluster of more than one defect (Eq. 8). This is why the defective probability increment is much smaller in case of 3:3 grouping ratio in comparison to $1: 1$ grouping ratio leading to higher yield in case of 3:3 grouping ratio. Finally, we observe that despite the yield loss due to clustering defects, it is still possible to reduce hardware cost (number of spare TSVs) by careful selection of grouping ratio and redundancy percentages. For example, from Table 2, it can be seen that $100 \%$ yield is possible for all defect distributions across all three sets of redundancy percentages. In case of $100 \%$ redundancy and grouping ratio of 4:4, 6,000 spare TSVs and 60,000 (2-to-1) multiplexers are needed to obtain $100 \%$ yield. The hardware cost in terms of spare TSVs can be reduced by using $50 \%$ redundancy and grouping ratio of $8: 4$, which achieves $100 \%$ yield using same number $(60,000)$ of multiplexers but with only 3,000 spare TSVs thereby saving 50\% spare TSVs without affecting targeted $(100 \%)$ yield. Moreover, additional spare TSV savings are possible by using $25 \%$ redundancy and grouping ratio of $20: 5$, but that comes at the cost of $(67,500-60,000)$ 7,500 additional multiplexers. These two experiments clearly demonstrate the trade-off between yield and hardware cost (number of multiplexer and spare TSVs) and show that careful selection of grouping ratio and redundancy percentage (spare TSV) can reduce the number of multiplexers and spare TSVs without affecting yield, when considering independent and clustering defect distributions.

\section{CONCLUSION}

TSV based 3D-ICs lead to low yield in current fabrication process. We propose a TSV grouping technique for allocating spare TSVs with regular ones in order to achieve highest possible yield at lowest possible hardware cost (number of multiplexers and spare TSVs) under independent and clustering defect distributions. To the best of our knowledge, this is the first study to propose a modelling mechanism for clustering defects on TSVs, furthermore it shows how yield can be calculated using clustering defect distribution. Simulation results show that for a given number of regular TSVs and failure rate, it is possible to achieve $100 \%$ yield while minimizing hardware cost (number of multiplexers and spare TSVs) both under independent and clustering defect distributions. This is achieved through careful selection of grouping ratios and redundancy percentage of spare TSVs.

\section{ACKNOWLEDGEMENTS}

This work is supported in part by EPSRC (UK) under grant no. EP/H011420/1. The authors would like to thank E. J. Marinissen for useful discussions.

\section{REFERENCES}

[1] K. Banerjee et al., "3-D ICs: A novel chip design for improving deepsub-micrometer interconnect performance and systems-on-chip integration", Proc. IEEE, vol. 89, pp.602 - 633, 2001.

[2] A. Topol et al., "Enabling SOI based assembly technology for threedimensional (3-D) integrated circuits (ICs)", IEDM, p.363, 2005.

[3] B. Swinnen et al., "3D integration by $\mathrm{Cu}-\mathrm{Cu}$ thermo-compression bonding of extremely thinned bulk Si die containing $10 \mathrm{~m}$ pitch through Si vias", Proc. IEDM Conference, December 11-13, 2006

[4] R. Reif et al., "Fabrication technologies for three-dimensional integrated circuits," Proc. ISQED, p.33, 2002.

[5] V. Suntharalingam, et al., "Megapixel CMOS image sensor fabricated in three-dimensional integrated circuit technology", Proc. IEEE Int. SolidState Circuits Conf. Tech. Dig., p.356, 2005.

[6] N. Miyakawa, et at., "Multilayer stacking technology using wafer-towafer stacked method," ACM, JETC., vol. 4, no. 4, 2008.

[7] L. Jiang, et al., "Yield enhancement for 3D-stacked memory by redundancy sharing across dies," ICCAD, pp.230-234, 2010.

[8] R. Nain et al., "Yield improvement of 3D ICs in the presence of defects in through signal vias," ISQED, pp.598-605, 2010.

[9] I. Loi, et al., "A low-overhead fault tolerance scheme for TSV-based 3 D network on chip links", ICCAD, pp.598 - 602, 2008.

[10] A.-C. Hsieh, et al., "TSV Redundancy: Architecture and Design Issues in 3D IC," DATE 2010, pp. 166-171.

[11] D. Velenis, et al., "Cost Effectiveness of 3D Integration Options," 3 DIC'10, pp.1-6, 2010.

[12] U. Kang et al., "8Gb 3D DDR3 DRAM Using Through-Silicon-Via Technology," ISSCC, pp.130-131, 2010.

[13] D. J. Friedman et al., "Model-free estimation of defect clustering in integrated circuit fabrication", IEEE TSM, vol. 10, pp.344 - 359, 1997.

[14] C. H. Stapper, "On yield, fault distributions, and clustering of particles," IBM Journal of Research and Development, pp.326-338, 1986.

[15] E. J. Marinissen and Y. Zorian, "Testing 3D chips containing through silicon vias," in International test conference, 2009.

[16] T. Fukushima, et al., "New Three-Dimensional integration Technology Based on Reconfigured Wafer-on-Wafer Bonding Technique".IEEEIEDM , pp. 985-988, 2007.

[17] C. H. Stapper, F. M. Armstrong, and K. Saji, "Integrated circuit yield statistics," Proc. IEEE, vol. 71, pp.453 - 470, 1983.

[18] M. Tahoori, "Defects, yield, and design in sublithographic nanoelectronics," DFT'05, pp. 3-11.

[19] F. J. Meyer and D. K. Pradham, "Modeling defect spatial distribution," IEEE Trans. Comput., vol. 38, pp.538 - 546, 1989.

[20] I. Koren et al., "A unified negative binomial distribution for yield analysis of defect tolerant circuits", IEEE Trans. Comp., vol. 42, 1993.

[21] A. Tyagi and M. A. Bayoumi, "Defect clustering viewed through generalized poisson distribution", IEEE TSM, vol. 5, pp.196-206, 1992. 